\title{
Enhancing elementary science learning through organic chemistry modeling and visualization
}

\author{
Daniel B. Fried ${ }^{1}$, Pablo P.L. Tinio ${ }^{2}$, Aaron Gubi ${ }^{3}$, and Jean P. Gaffney ${ }^{4,5}$ \\ ${ }^{1}$ Department of Chemistry, Saint Peter's University, Jersey City, New Jersey, USA \\ 2Department of Educational Foundations, Montclair State University, Montclair, New Jersey, USA \\ ${ }^{3}$ Department of Advanced Studies in Psychology, Kean University, Union, New Jersey, USA \\ ${ }^{4}$ Department of Natural Sciences, Baruch College City University of New York, New York, New York, 5 USA \\ ${ }^{5}$ The Graduate ${ }^{5}$ Center Program in Biology, City University of New York, New York, New York, USA \\ For correspondence: dfried1@saintpeters.edu
}

\begin{abstract}
The scientists of the future will need to begin studying science in great depth, much earlier in life, in order to keep up with the accelerating pace of discovery and technological innovation. For this to be possible, engaging and childfriendly pedagogical approaches need to be developed that leverage the natural enthusiasm of young science learners, as many students lose interest in science well before they encounter the rich subject matter of high school and college. This study provides evidence that a specially-designed chemistry curriculum can allow students 8-11 years old to rapidly learn advanced material while motivating them. The curriculum adhered to a backward design strategy and utilized a visual approach to chemistry learning. It also featured extensive use of hand-held organic chemistry model building and computerized molecular modeling activities. The 5-session study involved 63 students from grades 3-5 from an economically disadvantaged urban school in New Jersey. Despite the technical nature of the program, student motivation for chemistry learning was high, and assessment results showed students' high-level understanding of the material.
\end{abstract}

Keywords: Organic chemistry, Molecular modeling, Elementary

\section{Introduction}

Besides the possible long term academic benefits of providing a richer chemistry curriculum early in life, young students have a natural desire to ask what things are made of, and why living and nonliving things are the way they are (Clements and Sarama, 2016). While children enter school eager to learn, research demonstrates a gradual decline in children's motivation toward science as they advance through middle childhood and approach adolescence (Long, et al., 2007). This closing of the mind toward science experiences may prevent many children from fully developing scientific literacy, reducing their understanding with regards to science, technology, medicine, and environmental concerns, and limiting their vocational, socioeconomic, and overall life potential as adults (Metz, 2008; National Academy of Sciences, 2007; Nord, et al., 2011).

Research findings indicate that declines in motivation towards science education are not inevitable (Vedder-Weiss and Fortus, 2012). As a central scientific discipline, organic chemistry builds a sophisticated understanding of diverse subjects ranging from materials science to biochemistry. A positive experience in organic chemistry classes could therefore channel more students into STEM careers and could improve confidence in other science subjects. But how can organic chemistry, a college-level subject, be injected into an already crowded elementary curriculum? The study presented here involved a child-friendly organic chemistry program that consisted of five 45-minute 
class sessions that were given to elementary students. The course was designed to enrich any elementary science curriculum with minimal disruption to the standard curriculum. Important topics such as atomic structure and molecular bonding were efficiently and effectively taught through a unique visual approach to organic chemistry learning. Overall, the course provided a fresh and highly rewarding experience for the students, and importantly, gave them motivation and confidence in science because they were aware that they were studying college-level material.

This study used an extensive set of visualization tools to support student learning. Visualization is central to science learning, from rough sketches of the relationships amongst molecules and atoms to diagrams in chemistry textbooks ( $\mathrm{Wu}$ and Shah, 2004). Visualizations have been shown to improve modeling skills among all learners, especially those who are lower performing as compared to their peers (Dori and Kaberman, 2012; Kali and Linn, 2008). The custom-designed visualizations used in the study included animated presentations and hands-on model building activities that had been previously piloted in diverse schools and educational settings.

A major focus of this research program was to demonstrate that the science learning method is highly effective for all kinds of students, including those from disadvantaged backgrounds. To this end, a public elementary school in Jersey City, New Jersey with a diverse student body was selected to participate in the study. In New Jersey, the District Factor Group (DFG) is used to compare test scores in districts with similar demographics, and according to the New Jersey Department of Education, "represents an approximate measure of a community's relative socioeconomic status." The Jersey City School District scores in the second lowest of the eight District Ranking Groups. Demographics of the elementary school where the research was conducted are typical of the district as a whole.

\section{Methods}

\section{Participants}

The 63 students selected for the study were ages 8-11 and comprised three classes of equal size: a 3rd grade, a 4th grade, and a 5th grade. There were no special criteria for students to participate in the study; in fact, the classes comprised the entire roster of students from already-existing classes.

\section{Program Curriculum and Procedure}

All instruction was led by the first author, with the classroom teachers present, and consisted of five 45-minute class sessions for each grade. Five sessions were held, resulting in a total contact time with the students of approximately 3.5 hours. A pre-test was given at the beginning of the program to determine whether students had any familiarity with atoms and molecules. Students were also told that there would be an assessment in the final class that was very similar to a college chemistry test, and that they will probably be surprised by how much they will learn in the classes. The lessons of the program drew upon college chemistry curricula, particularly topics from general chemistry, organic chemistry, and biochemistry. Instruction relied on presentation slides that typically featured animations, color coding, simplified nomenclature, patterning, and other strategies to make complex materials accessible and exciting to young student learners. During the final meeting, a 15-minute written assessment was given to the students to evaluate their knowledge of the topics taught during the program.

The instructional methods used in the study were developed previously by the first author. The content of the program emphasized backward design in which the design of a lesson or curriculum begins with the identification of learning outcomes that then guide the lessons and activities. Backward design can enhance motivation and promote content learning among both exceptional and struggling learners (Childre, et.al., 2009), and relevant to this study, among science students (Spaulding and Flannagan, 2012). 
In the first class, students were presented with an interactive computer rendering of the crystal structure of the rhodopsin protein, the photo-sensitive molecule in the eye's retina. Students were encouraged to rotate, magnify, and explore the structure, and the class began to discuss patterns that they observed such as correlations between atom type and the number of bonds formed with other atoms, the presence of aromatic rings, and the abundance of hydrogen atoms. Students were told that the goal of the class was to learn why the atoms in the structure were bonded the way they were, and that by learning the material, they would get an idea of how the protein allows the eye to detect light.

Following this introduction, students began to learn about the periodic table. Students learned how to predict atomic structure, especially electron configuration, so that they could determine an element's covalent and hydrogen bonding tendencies. Students used this knowledge to draw organic chemistry structures and build them with molecular model kits. Throughout the program, students were introduced to the structure and properties of gas molecules, hydrocarbons, amino acids, and flavor molecules. Special emphasis was given to molecules and materials encountered in everyday life.

The program's focus on model building provided students with powerful insight into structure/function relationships and the diversity of chemical architectures. The hands-on aspect of the class at the outset, as well as the fact that the students were aware that they were learning "college-level" science, made the experience highly enjoyable and empowering. Finally, the curriculum featured 3D computer molecular modeling (PyMol, Schrödinger LLC), which allowed students to apply their knowledge of organic chemistry to biomolecules such as enzymes and DNA that would be too large to build with the handheld models. Altogether, the curriculum contrasts traditional science learning frameworks and is intended to go beyond current science standards. The impressive student results described below are the result of only 3.5 hours of instruction, so it is exciting to imagine what kinds of student achievement would be possible with a multi-year expansion of this curriculum.

During each class, students made chemical drawings and took notes on worksheets that matched the day's lessons. Each student used molecular modeling sets from Molymod, and a significant portion of each class was dedicated to their use. Individual and group model building exercises were used to teach students advanced concepts including molecular geometry, the concept of a chemical formula, and the meaning of a chemical isomer. Students also performed "hand-held chemical reactions" including acid/base reactions and dehydration reactions to make biopolymer models like peptides and polysaccharides. Students also took turns using the PyMol molecular modeling software to visualize the 3D structure of small organic molecules and biomolecules, particularly hemoglobin and rhodopsin, whose functions (oxygen transport and vision, respectively) were easy to grasp. By referring to proteins from the very first class, students could see how simple chemistry concepts applied to larger biochemistry structures, giving more meaning to each topic. At the end of the program, a twelve-question assessment was given to test technical knowledge about chemical bonding.

Detailed description of lessons

All class sessions featured model building during the presentations, facilitating a fun and active learning classroom environment. Presentations featured animations, often based on structures created in PyMol, as well as specialized child-friendly chemistry visualizations created in Keynote and ChemDraw.

Day 1: A brief pre-test asking students about their background knowledge of atoms and molecules was given. As instruction began, students learned about the difference in scale between atoms, molecules, and cells, using visual presentations. Students learned the color coding scheme of atoms important in biochemistry and practiced identifying them with plastic models and $3 \mathrm{D}$ computer 
models. The idea of the atomic and molecular nature of matter was introduced by allowing students to build and handle models of several solids, liquids, and gasses.

Day 2: Students continued building using the Molymod kits, constructing small molecules such as methane and other hydrocarbons, ammonia, amines, water, and alcohols, and learned to write their formulas. After gaining familiarity with how atoms typically bond, students began building and drawing their own molecules that followed the bonding rules they observed. For example, students observed that carbon always made four bonds, so they could begin to draw more complex structures, always keeping this rule in mind.

Day 3: What makes elements different from one another, aside from their bonding habits, was explored, first by considering differences in nuclear size. Students correlated atomic number with the number of protons in an element, and handled specimens of aluminum, iron, copper, and tungsten to feel the impressive differences associated with increased density and atomic nucleus mass. Students then were introduced to the connection between an element's position on the periodic table and their valence electron arrangement. A specialized animated presentation was used to teach students to draw Lewis structures of the first ten elements of the periodic table.

Day 4:The concept of covalent bonding was introduced using specialized animations. Students built models and drew representations of carbon-rich molecules using a nomenclature similar to Lewis dot structures. The building exercise featured molecules responsible for fruit and spice flavors. For example, students could build a model of isoamyl acetate (banana flavor) to connect the idea of a bonded molecular structure with the experience of taste. The lesson culminated in a "hand held chemical reaction" in which the entire class worked together to polymerize models of ethylene into polyethylene, creating an impressive polymer that was about ten feet long.

Day 5: Students began to explore chemical diversity by building models of the 20 amino acids. The concept of acid and base was also introduced and students learned how to remove and attach labile protons to create charged or neutral conjugate acid and base structures. Students were then allowed to modify their amino acid side chains to create artificial amino acids, and then drew the molecule they had constructed. The class closed with a peptide building activity in which all the students connected their amino acids via dehydration reactions to form a giant polymer, simulating the activity of the ribosome. The assessment was given at the end of the class period.

\section{Results}

Pre-test

The goal of this study was to document chemistry content learning in 8-11-year-old students after receiving instruction in a specialized chemistry curriculum. A pre-test, given on the first day of the program, asked students to describe or name an example of an atom and a molecule.

About half of the 63 students did not provide any response to the pre-test prompts, leaving them blank. The remaining students mostly responded by writing that atoms were "small" or "tiny." Several students mentioned the words "explosion" or "poison." None of the 63 students could name a specific atom or molecule. Care was taken to not make students feel insecure that they had so little knowledge of chemistry, and the pre-test was treated as an informal brainstorming activity so that the students did not struggle excessively and lose motivation.

Post-test

The post-test was more elaborate than the pre-test because the students had not been exposed to organic chemistry previously; this was the issue that the current program was intended to address. 
The twelve questions from the post-test probed student understanding of basic organic chemistry concepts and were intended to show literacy of several chemistry nomenclatures. Figure 1 shows a completed assessment by a fifth grade student, for reference. Several questions required that students interpret the periodic table to deduce atomic structure and bonding behavior. Other questions, drawn directly from sophomore organic chemistry exams, required students to create legitimate chemical structures with or without formula prompts, or to correct improperly-drawn chemical structures. In summary, the knowledge and skills addressed in the post-test comprised the prerequisites for studying more advanced concepts in organic chemistry, materials science, and biochemistry.

Questions 1-3 required students to interpret a drawing similar to a Lewis dot structure, and determine what element it represented by noticing the number and arrangement of electrons. Students were then asked to complete the structure by adding hydrogen atoms to complete the "neon configuration" of electrons. Finally, they were asked to identify the structure they had drawn as a water molecule.

Questions 4-5 demonstrated student's familiarity with organic chemistry "carbon skeleton" structures, commonly used by scientists. Since the hydrogen atoms are not shown in such structures, students of chemistry must be able to visualize where they are. Question 4 asked students to add bonded hydrogen atoms to the vanillin molecule in the correct positions. Question 5 asked students to write the correct molecular formula for vanillin.

Questions 6 and 7 required students to find an error in the bonding of acetic acid. Students needed to correct an oxygen which was bonded to too many hydrogens. This correction was usually accomplished by removing two of the hydrogens.

Questions 8 and 9 were designed to directly show the progress students made from the pretest where students were asked to name an example of a molecule. These post-test questions asked students to draw the molecular structure of dimethylamine, based on its formula, and then to draw any molecule, without any formula prompt.

Finally, questions 10-12, labeled on the assessment as extra credit, required students to infer the bonding behavior of phosphorus, an element that was not mentioned at all during the 3.5 hour course. To answer the questions, the students needed to use the periodic table to draw an atom diagram with electrons placed in their proper orbitals. They then needed to use knowledge from the class to determine the connection between the orbital arrangement and bonding. Once the student realized that phosphorus should be capable of making three bonds, they could then draw a hypothetical phosphorus-containing molecule.

Figure 2 shows the percentage of students, by grade level, who answered each of the questions correctly. For example, $100 \%$ of the 3rd graders answered question 1 correctly, identifying the atom diagram as oxygen. Seven of the twelve questions were answered correctly by at least $50 \%$ of the fifth graders. And at least $25 \%$ of the fourth graders answered the challenging final three questions correctly. Only a small fraction of the students received full credit for Question 4, which involved locating all eight hydrogens on the skeleton structure of vanillin. However, all three grades averaged between $40 \%$ and $50 \%$ for that question when partial credit for adding some or most of the hydrogens was added. Overall about $75 \%$ of participants in the program answered question 6 correctly and properly identified the mistake in the structure of acetic acid. 

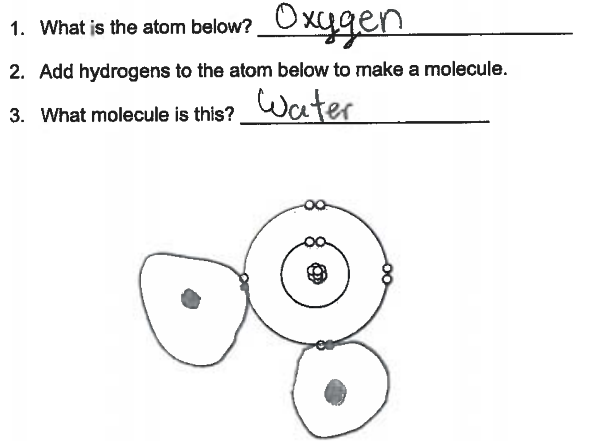

4. Add the missing hydrogens to complete the vanillin molecule below.<smiles>COc1cc(C=O)ccc1O</smiles>

5. Write the formula of vanillin. $\quad \mathrm{O}_{3} \mathrm{C}_{8} \mathrm{H}_{8}$

6. Circle the mistake in the molecule below.

7. Draw the corrected molecule in the box.
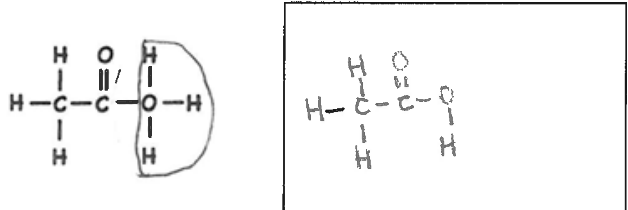

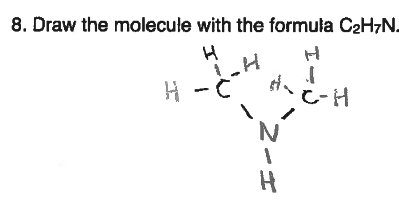

8. Draw the molecule with the formula $\mathrm{C}_{2} \mathrm{H}_{7} \mathrm{~N}$

$$
\text { I }
$$$$
H-C, H, C-H
$$

9. Make your own molecule and write the formula.

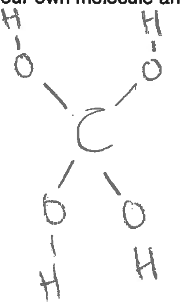

Extra credit!

Draw a phosphorus atom showing the protons, electrons, and orbitals.
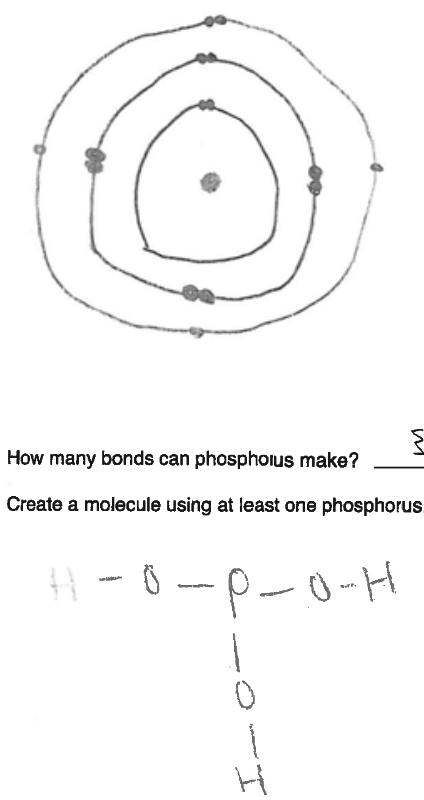

Figure 1. Sample final assessment completed by a fifth grade student.

A noteworthy finding is that several students demonstrated mastery of all of the basic concepts presented in the five-session program. These students understood how to interpret Lewis-dot structure atomic diagrams. They were also able to interpret organic chemistry structure drawings and create their own molecule drawings. Most impressive, these students were able to predict how a previously unknown element behaves in molecules. The data also reveals that the students were able to answer many technical questions about chemistry that they would have had no way of answering before the class.

Of special interest is the success of 4th and 5th graders on Questions 8 and 9. These questions gave students the opportunity to be creative, asking them to draw molecules first with a formula prompt, and then without a formula prompt. The fact that over half of the 4th and 5th grade students were able to draw technically correct molecules is noteworthy, especially given the fact that before the class, students could not clearly explain what a molecule was or name an example of one. 


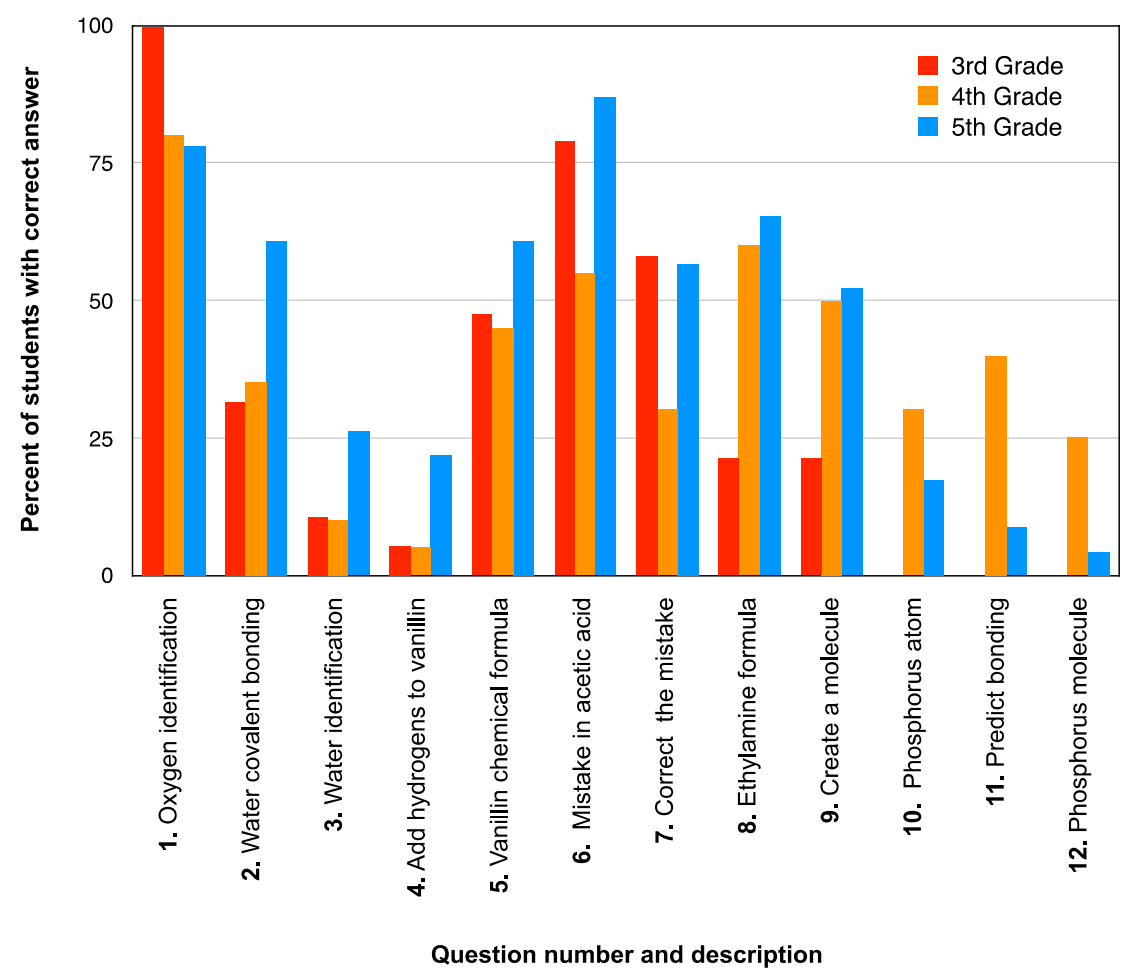

Students of all three grades achieved even higher success in Question 6, with over 75\% of 3rd and 5th graders able to correct a structure that was drawn incorrectly. The fact that students so young can look at an organic chemistry drawing and determine whether or not it is drawn correctly shows basic chemistry literacy, and is a skill that few students have, outside of those taking high school or college chemistry.

Fewer students succeeded in the very challenging Questions 10,11, and 12, which probed their ability to integrate knowledge of the periodic table to predict bonding patterns of an unfamiliar element. Although only $25 \%$ of fourth graders answered these correctly, it is still impressive given the short duration of the program and the very brief amount of time allotted for the assessment. That even some of these young students were able to correctly answer such high-level critical thinking questions shows much promise for this pedagogical approach.

\section{In-class Observations and Free Response Questions}

Despite its technical nature, the chemistry learning program was enthusiastically anticipated by the students each week, and they were highly engaged in the material. Classroom teachers reported that students continued the discussion of organic chemistry outside of the weekly classes, and several students even incorporated organic chemistry concepts into independent projects or into other science classroom assignments. Notably, some of the classroom teachers mentioned that several students who were thriving in the chemistry program had previously been struggling in the normal classroom science curriculum. Also, several of the most participatory and highest achieving students in the program were English language learners, who were described by their teachers as withdrawn or selfconscious before the program began. Teachers also commented that the highly visual and hands-on nature of the learning environment allowed students who may have had trouble engaging with more traditional curriculum, or with curriculum that is less visual, to succeed. By the time the program ended, teachers reported that several students had gained noticeable confidence in science and other 
subjects.

Generally, classes were fast-paced, and students were actively involved in the learning, and were especially eager to build more and more complex molecules with the molecular building kits. The desire to know what molecules make up everything in the world was obvious. From the beginning of the course, students began to ask thoughtful and even challenging questions about chemistry. Among the most engaging activities for the class were learning about and building flavor molecules, the polyethylene plastic polymer, and the peptide.

A post-class questionnaire asked students about what they liked learning most in the program, what it felt like to learn college-level material, and what other topics they would like to study in the future. The responses showed a basic understanding of what atoms and molecules were, which was lacking before the program, as revealed by the pre-test. Students were able to discuss what they learned and would like to learn in a coherent, scientifically literate manner. Generally, the responses showed an affinity for learning about the molecular nature of things, and students loved building molecules to understand the molecular structures of everyday materials. Perhaps even more important than the content the students absorbed is that the students gained an enthusiasm for chemistry, a feeling of self-confidence in science, and a desire to learn more.

On the questionnaire, one student wrote that they wanted to learn about "all the atoms that exits." Another student wanted to know whether flavor molecules could be combined. This is a relevant question, since many of our perceived flavors, for example coffee, chocolate, or meat, are in fact perceived from combinations of many molecules, and do not derive from a single flavor molecule.

Another student asked a wonderfully sophisticated question: "How do scientists figure out molecules?" This student wanted to connect with not just with the knowledge science has produced, but with the scientific process itself.

Many students expressed surprise and pride that they could learn college level science. One student wrote, "It felt like I was the smartest kid in the school. The science was easy to learn." Another wrote, "What it felt like to learn college science was awesome and weird. Yes I was surprised I could do it. It felt easy, in fact it was easy."

\section{Discussion}

A striking result from the study is not just that elementary students readily acquire organic chemistry knowledge, but that they are able to learn so quickly. In just 3.5 hours of instruction, many students advanced from minimal knowledge (e.g., knowing that atoms were small or "poison") to a level where they could competently predict the electron configurations and bonding behavior of atoms. The achievements of the students are even more impressive given the fact that the class met only once a week, and that no compulsory homework was given. One surprising result was how students answered assessment question 3. This question asked students to name the molecule they created in question 2, which was intended to be a water molecule, consisting of one oxygen and two hydrogens. One-third of the students stated that the molecule they created was not water, but neon. This answer, while incorrect, shows that students had internalized the idea of the neon configuration which oxygen indeed achieves through its bonding with the two hydrogen atoms. Responses like these will help guide future iterations of the program to improve student learning.

Other revealing effects of the program on students can be found in the written responses, like those exemplified by the question posted about "whether flavor molecules could be combined". This question could be interpreted as asking how modifying a flavor molecule would alter how we 
perceive it, or how mixing flavor molecules creates a new flavor. Such questions show how students have begun to connect chemical structure and diversity to everyday experiences, and would have been unimaginable before the student experienced the program, when the meaning of the word molecule was still obscure to them.

Throughout the study, students referred to 3D computer renderings of complex protein and DNA structures to practice the simpler organic chemistry concepts that they were learning in class. This allowed students to grasp the big picture about why they were learning about bond order and lonepair electrons. They became aware that everything they experience in everyday life, including living matter, was made of the same atoms that organize themselves in similar patterns. This appreciation for the scope and diversity of chemistry was apparent as the students created models and drew structures. They always wanted to know, "what molecule is this?"

Many of the responses to question 9, which prompted students simply to draw a molecule, were often elaborate, containing dozens of atoms which were properly or nearly-properly bonded into large, imaginative structures. The work put into these responses showed that students were excited by the prospect of building more and more complex molecules based on the rules they learned in the class. This child-like curiosity and drive to create and build within the limits governed by the laws of nature, are the qualities of great scientists, and were brought out by the curriculum. In summary, the curriculum successfully coupled high-level content with an engaging, child-friendly approach.

\section{Limitations}

In future studies, it is recommended that a larger treatment dosage be provided. Daily, rather than weekly instruction, would most likely result in even greater student achievement and more fully demonstrate the potential of this chemistry pedagogical approach. Lengthier duration of instruction would help show the extent to which elementary or middle school students could acquire the material. Furthermore, it is likely that the teaching approach developed for this study may be applicable to teaching other disciplines such as physics and biology. It is recommended that future education researchers seek to apply this method to related STEM courses and concepts. On a related note, future studies of this approach should seek to utilize a more rigorous pre/post design to better understand direct changes that occurred through the teaching intervention.

\section{Conclusion}

This study confirmed that elementary school students are capable of quickly learning organic chemistry topics that are normally reserved for high school or college courses, if immersed in the learning environment of the program. Given the extremely limited duration of this chemistry intervention ( 3.5 hours over five sessions) it must also be concluded that the program just scratches the surface of potential student achievement using these methods. This conclusion is strengthened when one considers that this 8-11-year-old cohort entered the study without substantial prior knowledge of chemistry. The realization that students could not only rapidly learn the high-level science material taught, but also demonstrate motivation and enthusiasm while doing so, will hopefully inspire new frameworks and opportunities for K-12 science learning.

\section{Acknowledgements}

We would like to thank Jersey City Public Schools and its faculty and administrators for welcoming us into their schools and allowing the project to take place. 


\section{References}

Childre, A., Sands, J. R., \& Pope, S. T. (2009). Backward Design. Teaching Exceptional Children, 41(5), 6-14.

Clements, D. H., \& Sarama, J. (2016). Math, science, and technology in the early grades. The Future of Children, 75-94.

Dori, Y. J., \& Kaberman, Z. (2012). Assessing high school chemistry students' modeling sub-skills in a computerized molecular modeling learning environment. Instructional Science, 40(1), 69-91. doi:10.1007/s11251-011-9172-7

Kali, Y., \& Linn, M. C. (2008). Designing effective visualizations for elementary school science. The elementary school journal, 109(2), 181-198.

Spaulding, L. S., \& Flannagan, J. S. (2012). DIS2ECT. Teaching Exceptional Children, 44(6), 6-14.

Long, J. F., Monoi, S., Harper, B., Knoblauch, D., \& Murphy, P. K. (2007). Academic motivation and achievement among urban adolescents. Urban Education, 42, 196-222.

Metz, K. E. (2008). Narrowing the gulf between the practices of science and the elementary school science classroom. The Elementary School Journal, 109(2), 138-161.

National Academy of Sciences, National Academy of Engineering, and Institute of Medicine, Rising above the gathering storm: energizing and employing America for a brighter economic future (Washington, DC: The National Academies Press, 2007), pp. 1-591.

Nord, C., Roey, S., Perkins, R., Lyons, M., Lemanski, N., Brown, J., \& Schuknecht, J. (2011). The Nation's Report Card [TM]: America's High School Graduates. Results of the 2009 NAEP High School Transcript Study. NCES 2011-462. National Center for Education Statistics.

PyMOL: The PyMOL Molecular Graphics System, Version 2.0 Schrödinger, LLC.

Vedder-Weiss, D., \& Fortus, D. (2012). Adolescents' declining motivation to learn science: A follow-up study. Journal of Research in Science Teaching, 49(9), 1057-1095. doi:10.1002/tea.21049

Wu, H. K., \& Shah, P. (2004). Exploring visuospatial thinking in chemistry learning. Science education, 88(3), 465-492. 